\title{
Rendimento de coalhada obtida a partir de leite fresco, resfriado e congelado de ovelhas da raça Lacaune e caracterização física do soro obtido
}

\author{
Curd yeld obtained from fresh, cooled and frozen Laucane sheep milk and \\ physical characteristics of the obtained whey
}

\author{
Luisa Wolker Fava ${ }^{\mathrm{I}}$ Irene Clemes Külkamp-Guerreiro ${ }^{\mathrm{II}}$ Andrea Troller Pinto ${ }^{\mathrm{I}}$
}

\section{RESUMO}

A composição do leite, que pode ser afetada pelo resfriamento ou congelamento, é determinante para o rendimento na fabricação de queijos, sendo um parâmetro muito importante em termos econômicos. O presente estudo teve como objetivo avaliar o efeito do resfriamento e do congelamento do leite, durante sete dias, sobre o rendimento da coalhada produzida a partir de leite de ovelha e sobre as características físicas do soro obtido. Entre janeiro e dezembro de 2011, foram coletadas mensalmente amostras de leite de ovelha, diretamente dos tanques de resfriamento. Uma parte foi submetida à análise no mesmo dia, denominada leite fresco, $e$ as restantes armazenadas até sete dias, uma em refrigeração $\left(5^{\circ} \mathrm{C}\right.$ - leite resfriado) e outra em congelamento doméstico $\left(-4^{\circ} \mathrm{C}\right.$ - leite congelado). Determinou-se o rendimento da coalhada e densidade, $\mathrm{pH}$ e acidez titulável do soro obtido. Verificou-se diferença significativa $(P<0,05)$ no rendimento da coalhada entre os tratamentos aplicados, sendo que o rendimento obtido com leite resfriado $\left(3,64 \pm 0,34 \mathrm{~L} \mathrm{~kg}^{-1}\right)$ e congelado $\left(3,18 \pm 0,23 \mathrm{~L} \mathrm{~kg}^{-1}\right)$ não apresentaram diferenças com o de leite fresco $\left(3,38 \pm 0,22 \mathrm{~L} \mathrm{~kg}^{-1}\right)$, porém estes tratamentos diferiram entre si. A densidade média do soro obtido $\left(1,025 \mathrm{~g} \mathrm{~mL}^{-1}\right)$ não apresentou diferenças entre os tratamentos aplicados. $\mathrm{O} p \mathrm{H}$ e acidez titulável do soro foram semelhantes nos tratamentos de leite fresco e congelado, havendo diferenças $(P<0,05)$ com o soro de leite resfriado, que apresentou-se mais ácido. Embora o soro da coalhada obtida a partir de leite resfriado apresente-se mais ácido, o resfriamento e o congelamento do leite ovino, por um período de sete dias, não afetam o rendimento na fabricação de queijos e o soro obtido não apresenta diferença de densidade entre os tratamentos aplicados.

Palavras-chave: rendimento de queijo, congelamento, soro de leite ovino.

\section{ABSTRACT}

Milk composition that can be affected by cooling or freezing is crucial for the performance of chesese making, and it is an important parameter in economic terms. The objective of this study was to evaluate, during 7 days, the effect of milk cooling and freezing on the yield of the curd produced with the sheep milk and on the physical characteristics of the obtained whey. Between January and December 2011 samples of the sheep milk were collected every month, directly from the bulk tanks. A part of it was subject to analysis in the same day, and the rest was stored for up to 7 days. One of the samples was kept under refrigeration $\left(5^{\circ} \mathrm{C}\right.$ - cooled milk) and another was kept in a domestic freezer $\left(-4^{\circ} \mathrm{C}-\right.$ frozen milk). The yield and density of the curd, $\mathrm{pH}$ and titratable acidity of the obtained whey was determined. The treatment applied showed a significant difference $(P<0.05)$ in the yield of the curd, whereas the yield obtained with the cooled milk $(3.64 \pm 0.34 \mathrm{~L}$ $\left.\mathrm{kg}^{-1}\right)$ and the frozen milk $\left(3.18 \pm 0.23 \mathrm{~L} \mathrm{~kg}^{-1}\right)$ showed no differences with the fresh milk $\left(3.38 \pm 0.22 \mathrm{~L} \mathrm{~kg}^{-1}\right)$, but these treatments were not the same. The average density of the obtained whey $(1.025 \mathrm{~g}$ $\left.\mathrm{mL}^{-1}\right)$ did not present differences between the applied treatments. The whey $\mathrm{pH}$ and titratable acidity were similar in both fresh and frozen milk treatments, but there was a difference $(P<0.05)$ with the cooled milk, which had a higher acidity. Even though the whey of the curb obtained from the cooled milk is more acid, the cooling and freezing of the sheep milk, for a period of 7 days, do not affect the yield in cheese making, and the obtained whey does not present density differences between the applied treatments.

Key words: cheese yield, sheep milk whey, freeze.

\section{INTRODUÇÃO}

O leite de ovelha caracteriza-se, principalmente, por sua riqueza em sólidos, sendo raramente consumido na forma fluida $\mathrm{e}$ amplamente utilizado na fabricação de queijos (RAMOS; JUAREZ, 2011). A composição do leite,

'Departamento de Medicina Preventiva, Faculdade de Medicina Veterinária, Universidade Federal do Rio Grande do Sul (UFRGS), Av. Bento Gonçalves, 9090, 91540-000, Porto Alegre, RS, Brasil. E-mail: andrea.troller@ufrgs.br. *Autor para correspondência.

IIDepartamento de Produção de Matéria-prima, Faculdade de Farmácia, UFRGS, Porto Alegre, RS, Brasil.

$$
\begin{array}{cc}
\text { Recebido 20.05.13 Aprovado 07.11.13 Devolvido pelo autor 14.02.14 } \\
\text { CR-2013-0708.R1 }
\end{array}
$$


principalmente seu teor de proteína, é um importante fator para a produção de queijos, por ser determinante no rendimento e na qualidade do produto final (NGKWAI-HANG et al., 1982).

A utilização de leite congelado para a produção de queijos, no período de entressafra, pode ser uma alternativa para solucionar a sazonalidade da produção leiteira (KATILI et al., 2006). Contudo, a velocidade do congelamento pode afetar a qualidade do produto final, sendo que, no congelamento lento, ocorre a degradação de proteínas, acarretando problemas na formação da coalhada (BERGER, 2001). Além disso, o processo de congelamento apenas retarda a multiplicação de microrganismos (PORCIONATO et al., 2008), sendo de extrema importância a qualidade da matéria-prima para evitar defeitos nos queijos produzidos.

O resfriamento do leite por longos períodos pode causar a diminuição do fosfato de cálcio micelar, alterando a estabilidade proteica (WALSTRA et al., 2006) e afetando a formação da coalhada. Além disso, há seleção de microrganismos psicrotróficos, com a consequente produção de proteases, que podem influenciar na qualidade e no rendimento dos queijos, pois causam perdas de nitrogênio no soro e diminuição do peso do queijo produzido (MIRANDA; GRIPON, 1986). Essas alterações das características físico-químicas e do comportamento dos componentes do leite, juntamente com a seleção de microrganismos específicos, podem influenciar na qualidade e no rendimento dos queijos fabricados (BANKS et al., 1988).

O soro é o líquido resultante do processo de elaboração de queijos, a partir da separação da gordura e das caseínas do leite. Sua composição compreende mais da metade dos sólidos presentes no leite integral, incluindo a maior parte da lactose e $20 \%$ das proteínas, as quais possuem excelente qualidade. Antigamente, era considerado um resíduo inaproveitável das indústrias de laticínios, contudo, com o conhecimento de suas características físicoquímicas e com os avanços tecnológicos, atualmente, o soro possui grande valor para as indústrias alimentícia e farmacêutica (ORDÓÑEZ, 2005).

$\mathrm{O}$ rendimento pode ser definido como o peso do queijo, expresso em quilogramas $(\mathrm{kg})$, obtido a partir de $100 \mathrm{~kg}$ de leite. Trata-se de um parâmetro muito importante, pois, quanto maior o peso do queijo, maior é o ganho em termos econômicos (PAOLO et al., 2008). O rendimento do queijo varia de acordo com inúmeros fatores, incluindo a forma de estocagem da matéria-prima. O presente estudo teve como objetivo avaliar o efeito do resfriamento e do congelamento do leite, durante sete dias, sobre o rendimento da coalhada produzida a partir de leite de ovelha e sobre as características físicas do soro obtido.

O presente trabalho teve por objetivo iniciar pesquisas referentes à capacidade de produção queijeira do leite ovino congelado e resfriado, com base na capacidade de manter seu rendimento, quando comparado ao leite fresco, bem como sobre as características físico-químicas do soro.

\section{MATERIAL E MÉTODOS}

O presente trabalho foi realizado entre janeiro e dezembro de 2011. Mensalmente, foram coletadas amostras de leite de conjunto, de quatro propriedades produtoras de leite de ovelha, localizadas no município de Bento Gonçalves, Rio Grande do Sul, Brasil, cuja altitude média é de $600 \mathrm{~m}$ acima do nível do mar, com localização geográfica $29^{\circ} 08^{\prime} 47^{\prime \prime}$ de latitude sul e $51^{\circ} 31^{\prime} 17^{\prime \prime}$ de longitude oeste. A temperatura anual média é de $16,5^{\circ} \mathrm{C}$ e a precipitação é de $1823 \mathrm{~mm}^{a^{-1}}$ (BRITO et al., 2006). O clima temperado da região caracteriza-se pela homogeneidade pluviométrica, com alterações térmicas, possuindo semelhanças com a Europa e relacionadas ao clima e ao relevo (VIEIRA et al., 2007).

As coletas de leite foram realizadas diretamente do local de armazenamento e resfriamento do leite, sendo em tanque de expansão em uma propriedade e tanque de imersão em três e as amostras encaminhadas ao laboratório de Inspeção e Tecnologia de Leite e Derivados, Ovos e Mel em caixas isotérmicas com gelo.

Cada amostra das quatro propriedades foi dividida em três alíquotas: a primeira foi submetida à análise no mesmo dia, denominada leite fresco (LF), e as restantes armazenadas por sete dias, uma em refrigeração a $5^{\circ} \mathrm{C}$ (leite resfriado - LR) e outra em congelamento doméstico a $-5^{\circ} \mathrm{C}$ (leite congelado - LC). As amostras de leite congelado sofriam descongelamento lento por 24 horas, em refrigeração $\left(5^{\circ} \mathrm{C}\right)$, do sexto para o sétimo dia. Frações das alíquotas de leite fresco, resfriado e congelado foram encaminhadas para determinação dos teores de gordura, proteína, lactose e sólidos por espectroscopia de infravermelho, a laboratório de referência credenciado pela Rede Brasileira de Qualidade do Leite, segundo BRASIL(2002).

Determinou-se o rendimento da coalhada obtida com cada uma das amostras compostas de leite fresco, resfriado e congelado. As amostras compostas foram feitas misturando-se volumes iguais de leite, 
provenientes de cada propriedade (totalizando $1000 \mathrm{~mL}$ de leite). O leite não sofreu processo de pasteurização. A coalhada foi produzida mantendose o leite a $37^{\circ} \mathrm{C}$ e adicionando-se a ele $0,02 \mathrm{~g}$ de cloreto de cálcio por litro de leite (BEHMER, 1980) e $0,8 \mathrm{~mL}$ de coalho por litro de leite $\left(\mathrm{Chr}\right.$. Hansen $\left.{ }^{\circledR}\right)$. Esperava-se coagular por 30 minutos, quando era procedido o corte da coalhada em pequenos grumos (em torno de $1 \mathrm{~cm}$ de lado). A coalhada foi colhida com peneira fina e enformada em formas redondas de $10,5 \mathrm{~cm}$ de altura e $11 \mathrm{~cm}$ de diâmetro. O volume do soro resultante foi medido em proveta e realizouse análise de determinação da densidade a $20^{\circ} \mathrm{C}$ por termolactodensímetro, $\mathrm{pH}$, e acidez titulável em graus Dornic, de acordo com a legislação vigente (BRASIL, 2006).

Para expressar o volume total de soro eliminado no processo, dividiu-se o total do soro pelo volume total de leite utilizado na produção da coalhada. As coalhadas foram colocadas sem peso sob refrigeração $\left(5^{\circ} \mathrm{C}\right)$ durante $24 \pm 4$ horas, quando foi medido o peso da coalhada e o volume de soro residual. $\mathrm{O}$ rendimento foi calculado dividindo-se o volume de leite empregado no processo (litros) pela massa da coalhada obtida (quilogramas) (SILVEIRA; ABREU, 2003). Ainda, 24 horas após a produção das coalhadas, foi determinada sua umidade, utilizandose balança determinadora de umidade, modelo ID-50, segundo AOAC (1995).

No total, foram produzidas 36 coalhadas, sendo 12 para cada um dos tratamentos térmicos aplicados ao leite.Foi realizada análise estatística descritiva e os valores de rendimento foram submetidos à análise de variância (ANOVA), e as comparações entre os tratamentos foram realizadas utilizando-se o teste de Tukey $(\mathrm{P}<0,05)$. Utilizou-se o software SPSS 19.0 (SPSS Inc., Chicago, IL).

\section{RESULTADOS E DISCUSSÃO}

A caracterização físico-química do leite fresco, resfriado e congelado está apresentada na tabela 1. O resfriamento ou congelamento do leite por sete dias não alterou sua composição de forma significativa $(\mathrm{P}<0,05) . \mathrm{O}$ rendimento médio da coalhada e os valores máximos e mínimos obtidos em cada tratamento estão apresentados na tabela 2 . Houve diferença significativa $(\mathrm{P}<0,05)$ entre os tratamentos aplicados (LF, LR, LC).

Segundo ZHANG et al. (2006), o congelamento pode afetar o rendimento na fabricação de queijos, com redução significativa quando o leite é congelado por um período de seis meses. Neste mesmo estudo, o rendimento não foi afetado quando o congelamento não foi superior a dois meses, sofrendo moderada redução com três meses de congelamento. Da mesma forma, no presente estudo, com congelamento do leite por sete dias, a coalhada produzida a partir de leite congelado não apresentou diferença significativa com o rendimento da produzida a partir de leite fresco, porém apresentou tendência a ser menor.

Durante o congelamento, ocorre redução no conteúdo de gordura do leite, devido à formação de cristais de gelo que danificam os glóbulos, causando liberação de lipoproteínas da membrana, com consequente perda da estabilidade (ZHANG et al., 2006). Além disso, ocorre alteração da estrutura das proteínas, devido à destruição das pontes de hidrogênio entre os polipeptídeos, diminuindo a capacidade de retenção de água (FONTECHA et al., 1993). Contudo, apesar de diferenças físico-químicas entre o leite fresco e o leite congelado serem os principais fatores responsáveis por diferenças no rendimento de queijos (JAEGGI et al., 2004), no presente estudo, a coalhada produzida a partir de leite congelado obteve rendimento estatisticamente semelhante à coalhada de leite fresco $(\mathrm{P}<0,05)$.

$\mathrm{O}$ rendimento da coalhada produzida com leite fresco não diferiu dos rendimentos obtidos com leite resfriado ou congelado. Entretanto, essas duas últimas diferiram entre si $(\mathrm{P}<0,05)$, sendo necessária menor quantidade de leite congelado para produzir um quilograma de coalhada. Não existem estudos comparando o rendimento de queijos a partir de leite submetido a estes dois tratamentos, contudo esta diferença pode ter ocorrido devido a maiores alterações nas estruturas dos glóbulos de gordura e das micelas de caseína durante o resfriamento prolongado, já que estes são os principais componentes que afetam o rendimento (JAEGGI et al., 2004). Segundo WALSTRA et al. (2006), o resfriamento do leite, por períodos superiores a 24 horas, promove a desintegração das micelas de caseína, devido à dissolução de uma parte considerável da $\beta$-caseína e de parte do fosfato de cálcio micelar. Porém, o aquecimento subsequente do leite restabelece esta estrutura, mas é questionável se as micelas de caseína tornam-se idênticas à forma original. Além disso, o resfriamento promove a perda de alguns componentes da membrana do glóbulo de gordura, alterando de forma irreversível sua estrutura.

As porcentagens médias de soro eliminado, densidade, $\mathrm{pH}$ e acidez titulável do soro obtido a partir das coalhadas de leite fresco (LF), leite resfriado (LR) e leite congelado (LC) estão apresentados na 
Tabela 1 - Média e desvio padrão dos teores de gordura, proteína, lactose e sólidos totais do leite de mistura fresco (LF), resfriado (LR) e congelado (LC) de ovelhas da raça Lacaune, na região Serrana do Rio Grande do Sul/Brasil, no período de janeiro a dezembro de 2011 .

\begin{tabular}{lcccc}
\hline Tratamento & $\mathrm{n}$ & Gordura & Proteína & Lactose \\
\hline LF & 12 & $8,28 \pm 0,48$ & $5,27 \pm 0,20$ & $4,44 \pm 0,18$ \\
LR & 12 & $7,99 \pm 0,49$ & $5,25 \pm 0,26$ & $4,38 \pm 0,12$ \\
LC & 12 & $8,15 \pm 0,51$ & $5,26 \pm 0,22$ & $4,44 \pm 0,16$ \\
\hline
\end{tabular}

tabela 3. Houve diferença significativa $(\mathrm{P}<0,05)$ entre os tratamentos aplicados ao leite (LF, LR, LC) para os valores de $\mathrm{pH}$ e acidez titulável do soro obtido.

A densidade não apresentou diferenças entre os tratamentos aplicados, obtendo média de $1,025 \mathrm{~g} \mathrm{~mL}$. Segundo PHILIPPOPOULOS \& PAPADAKIS (2001), a produção de soro, em volume, apresenta variação sazonal e a composição varia ao longo do período de lactação. Não foram encontrados dados científicos que apresentem diferenças de densidade do soro quando o leite sofre tratamentos térmicos de refrigeração e congelamento.

$\mathrm{O} \mathrm{pH}$ e a acidez titulável do soro foram semelhantes nos tratamentos de leite fresco e congelado. O soro da coalhada, obtida a partir de leite resfriado, apresentou-se mais ácido, indicando maior acidez da matéria-prima utilizada no processo, causada por possível contaminação bacteriana do leite e multiplicação dos microrganismos durante o processo de refrigeração. A diminuição do $\mathrm{pH}$ do leite favorece a dissolução do fosfato coloidal, resultando em ligações mais fracas entre as caseínas (WALSTRA et al., 2006). Essa alteração nas micelas de caseína pode ser a causa da maior eliminação de soro da coalhada, obtida a partir de leite resfriado, devido à diminuição da capacidade de retenção de água.

O leite de cabra apresenta densidade e acidez titulável inferiores, quando comparado ao leite de ovelha (AMIGO \& FONTECHA, 2011; RAMOS \& JUAREZ, 2011). Apesar disso, em estudo realizado por NETO et al. (2006), o soro pasteurizado e resfriado, obtido a partir de queijo coalho de leite de cabra, apresentou-se mais ácido e com densidade superior ao encontrado no presente estudo, com

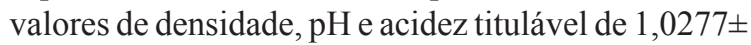
$0,0006 \mathrm{~g} \mathrm{~mL}^{-1}, 5,63 \pm 0,27$ e $18 \pm 2^{\circ} \mathrm{D}$, respectivamente. Apesar de não haver diferenças nas características físicas entre os soros provenientes das amostras de leite fresco e congelado, a coalhada obtida a partir de leite congelado apresentou maior retenção de soro, quando comparada às amostras de leite fresco e resfriado, eliminando menor volume ao final do processo. ZHANG et al. (2006), ao analisarem o rendimento do queijo Tipo Cheddar, obtido a partir de leite de ovelha fresco e congelado, observaram redução significativa no rendimento em amostras de leite congeladas por um período de seis meses. A redução no rendimento foi atribuída à diminuição da capacidade de retenção de água, causada pela alteração da estrutura das proteínas durante o processo de congelamento. Contudo, o rendimento não foi afetado quando as amostras de leite foram submetidas ao congelamento por um período de até dois meses, corroborando os resultados do presente estudo.

A composição físico-química do soro varia de acordo com o tipo de leite utilizado, com o tipo de queijo a ser produzido, com o tratamento aplicado e com outros processos efetuados durante a fabricação do queijo (ORRÙ \& PILLONI, 2008).

A caracterização da coalhada quanto ao seu teor de umidade está descrita na tabela 4. Coalhadas produzidas com o leite fresco e com o

Tabela 2 - Média e valores máximos e mínimos de rendimento da coalhada $\left(\mathrm{LKg}^{-1}\right)$, produzida a partir de leite fresco (LF), leite resfriado (LR) e leite congelado (LC) de ovelhas na raça Lacaune, na região Serrana do Rio Grande do Sul/Brasil, no período de janeiro a dezembro de 2011.

\begin{tabular}{|c|c|c|c|c|}
\hline Tratamento & $\mathrm{n}$ & Média & Máximo & Mínimo \\
\hline LF & 12 & $3,38 \pm 0,22^{\mathrm{ab}}$ & 3,83 & 3,02 \\
\hline LR & 12 & $3,64 \pm 0,34^{\mathrm{b}}$ & 4,27 & 3,14 \\
\hline LC & 12 & $3,18 \pm 0,23^{\mathrm{a}}$ & 3,71 & 2,86 \\
\hline
\end{tabular}

Médias seguidas pela mesma letra, na mesma coluna, não diferem entre si $(\alpha=0,05)$. 
Tabela 3 - Porcentagens médias de soro eliminado, densidade, pH e acidez titulável (AT) do soro, obtido a partir das coalhadas de leite fresco (LF), leite resfriado (LR) e leite congelado (LC) de ovelhas na raça Lacaune, na região Serrana do Rio Grande do Sul/Brasil, no período de janeiro a dezembro de 2011.

\begin{tabular}{lccccc}
\hline Tratamento & $\mathrm{n}$ & Soro eliminado $(\%)$ & Densidade $\left(\mathrm{g} \mathrm{mL}^{-1}\right)$ & $\mathrm{pH}$ & AT $\left({ }^{\circ} \mathrm{D}\right)$ \\
\hline LF & 12 & $67,89 \pm 2,27^{\mathrm{a}}$ & $1,0251 \pm 0,0010^{\mathrm{a}}$ & $6,52 \pm 0,12^{\mathrm{a}}$ & $14,87 \pm 1,14^{\mathrm{a}}$ \\
LR & 12 & $65,81 \pm 3,68^{\mathrm{a}}$ & $1,0246 \pm 0,0011^{\mathrm{a}}$ & $6,34 \pm 0,29^{\mathrm{b}}$ & $19,22 \pm 2,90^{\mathrm{b}}$ \\
LC & 12 & $61,02 \pm 3,05^{\mathrm{b}}$ & $1,0252 \pm 0,0011^{\mathrm{a}}$ & $6,57 \pm 0,15^{\mathrm{a}}$ & $14,80 \pm 0,99^{\mathrm{a}}$ \\
\hline
\end{tabular}

Médias seguidas pela mesma letra, na mesma coluna, não diferem entre si $(\alpha=0,05)$.

leite refrigerado não apresentaram teores de umidade estatisticamente diferentes $(\mathrm{P}<0,05)$. Entretanto, elas apresentaram-se significativamente diferentes das coalhadas produzidas com leite congelado. Esse resultado confirma o achado descrito anteriormente, já que houve maior retenção do soro nas coalhadas produzidas com leite congelado, traduzindo-se, assim, em uma maior umidade do produto final.

\section{CONCLUSÃO}

Os resultados do presente estudo demonstraram que o resfriamento e o congelamento do leite ovino, por um período de sete dias, não afetaram o rendimento na fabricação de queijos. Contudo, a coalhada produzida com leite congelado obteve melhor rendimento que aquela produzida com leite resfriado.

A densidade dos soros obtidos a partir do leite fresco, resfriado e congelado não apresentou variação. Porém, o soro da coalhada de leite resfriado apresentou-se mais ácido, o que pode dificultar sua utilização por parte das indústrias para a produção de derivados lácteos. O congelamento do leite não afetou as características físicas do soro obtido, permitindo sua utilização ao final do processo.

Os resultados obtidos indicam a necessidade de pesquisas mais aprofundadas na área, com a determinação de outros parâmetros de

Tabela 4 - Umidade da coalhada produzida a partir de leite fresco (LF), leite resfriado (LR) e leite congelado (LC) de ovelhas na raça Lacaune, na região Serrana do Rio Grande do Sul/Brasil, no período de janeiro a dezembro de 2011.

\begin{tabular}{ccccc}
\hline \multirow{2}{*}{ Tratamento } & & & \multicolumn{3}{c}{-------Umidade da coalhada (\%)---------- } \\
& $\mathrm{n}$ & Média & Máximo & Mínimo \\
\hline \multirow{2}{*}{ LC } & \multirow{2}{*}{12} & $41,17 \pm 2,72^{\mathrm{a}}$ & 44,65 & 36,05 \\
& & $41,33 \pm 3,97^{\mathrm{a}}$ & 46,15 & 34,05 \\
& & $45,68 \pm 2,13^{\mathrm{b}}$ & 48,70 & 42,20 \\
\hline
\end{tabular}

Médias seguidas pela mesma letra, na mesma coluna, não diferem entre si $(\alpha=0,05)$. rendimento queijeiro, tais como teores de proteína, gordura e matéria seca, entre outros.

\section{REFERÊNCIAS}

AMIGO, L.; FONTECHA, J. Goat milk. In: FUQUAY, J.W. et al. Encyclopedia of dairy sciences. 2.ed. United Kingdon: ELSEVIER, 2011. V.3, p.484-493.

AOAC (ASSOCIATION OF OFFICIAL AGRICULTURAL CHEMISTS). Official Methods of Analysis of the Association of Official Agricultural Chemists. 11.ed. Washington, 1995. 1015p.

BANKS, J.M., GRIFFITHS, M.W., PHILLIPS, J.D., MUIR, D.D. A comparison of the effects of storage of raw milk at $2{ }^{\circ} \mathrm{C}$ and $6^{\circ} \mathrm{C}$ on the yield and quality of Cheddar cheese. Food Microbilogy, v.5, n.1, p.9-16, 1988. Disponível em: <http: // dx.doi.org/10.1016/0740-0020(88)90003-2>. Acesso em: 08 jan. 2013. doi: $10.1016 / 0740-0020(88) 90003-2$

BEHMER, M.L.A. Tecnologia do leite. 10.ed. São Paulo: Nobel, 1980. $322 \mathrm{p}$.

BERGER, Y.M. Milking equipment for dairy ewes. In: DAIRY SHEEP SYMPOSIUM, 7., 2001, Eau Claire, Wisconsin. Anais Proceedings of the $7^{\text {th }}$ Great Lakes Dairy Sheep Symposium. Eau Claire: Wisconsin Sheep Dairy Cooperative, Strum, Wisconsin, USA, 2001. p.9-16.

BRASIL. Ministério da Agricultura, Pecuária e Abastecimento. Instrução Normativa n.68 de 12 de dezembro de 2006. Diário Oficial da União, 14 dez. Seção 1, p.8.

BRASIL. Ministério da Agricultura Pecuária e Abastecimento. Instrução Normativa n.37 de 18 de abril de 2002. Disponível em: <http://www.agricultura.gov.br>. Acesso em: 12 out. 2013.

BRITO, M.A. et al. Composição do sangue e do leite em ovinos leiteiros do sul do Brasil: variações na gestação e na lactação. Ciência Rural, v.36, p.942-948, 2006. Disponível em: <http:// dx.doi.org/10.1590/S0103-84782006000300033>. Acesso em: 20 out. 2012. doi: 10.1590/S0103-84782006000300033.

FONTECHA, J. et al. Infrared and Raman spectroscopic study of casein in cheese: effect of freezing and frozen storage. Journal of Dairy Science, v.76, p.3303-3309, 1993. Disponível em: $<\mathrm{http}: / /$ dx.doi.org/10.3168/jds.S0022-0302(93)77667-5>. Acesso em: 08 jan. 2013. doi: 10.3168/jds.S0022-0302(93)77667-5.

JAEGGI, J.J. et al. Milk composition and cheese yield from hard and soft cheese manufactured from sheep milk. In: DAIRY 
SHEEP SYMPOSIUM, 10., 2004, Hudson, Wisconsin. Anais Proceedings of the $10^{\text {th }}$ Annual Great Lakes Dairy Sheep Symposium. Hudson, Wisconsin: Dairy Sheep Association of North America, 2004. p.132-142

KATILI, L.M. et al. Aspectos físico-químicos e microbianos do queijo maturado por mofo obtido da coagulação mista com leite de cabra congelado e coalhada congelada. Ciência e Tecnologia dos Alimentos, v.26, p.740-743, 2006. Disponível em: <http:// dx.doi.org/10.1590/S0101-20612006000400004. Acesso em: 10 set. 2012. doi: 10.1590/S0101-20612006000400004.

MIRANDA, G.; GRIPON, J.C. Origine, nature et incidences technologiques de la protéolyse dans le lait. Le Lait, v.66, p.1-18, 1986.

NETO, B.A.M. et al. Caracterização do soro de leite de cabra utilizado na formulação de pão de forma. In: JORNADA NACIONAL DA AGROINDÚSTRIA, 2006, Bananeiras, Paraíba. Anais... Bananeiras: Universidade Federal da Paraíba, 2006. p.1-6.

NG-KWAI-HANG, K.F. et al. Environmental influences on protein content and composition of bovine milk. Journal of Dairy Science, v.65, p.1993-1998, 1982. Disponível em: <http:// dx.doi.org/10.3168/jds.S0022-0302(82)82449-1>. Acesso em: 08 jan. 2013. doi: 10.3168/jds.S0022-0302(82)82449-1.

ORDÓÑEZ, J.A. Tecnologia de alimentos - alimentos de origem animal. Porto Alegre: Artmed, 2005. V.2,279p.

ORRÙ, P.F.; PILLONI, M.T. Technical and economic analysis for the recovery of high biological value products from the cheese-making whey. International Journal of Mechanics and Control, v.9, p.4759, 2008. Disponível em: <http://www.jomac.it/FILES\%20RIVISTA/ JoMaC08A/JoMaC08A.pdf\#page=49>. Acesso em: 08 jan. 2013.

PAOLO, F. et al. Cheese yield: Factors of variation and predictive formulas. A review focused particularly on Grana type cheeses. Annali Facoltà di Medicina Veterinaria di Parma, v.28, p.211-
232, 2008. Disponível em: <http://www.unipr.it/arpa/facvet/ annali/2008/18\%20211_232.pdf>. Acesso em: 08 jan. 2013.

PHILIPPOPOULOS, C.D.; PAPADAKIS, M.T. Current trends in whey processing and utilization in Greece. International Journal of Dairy Technology, v.54, p.14-19, 2001. Disponível em: $<$ http://onlinelibrary. wiley.com/doi/10.1046/j.1471-0307.2001.00007.x/full>. Acesso em: 08 jan. 2013. doi: 10.1046/j.1471-0307.2001.00007.x.

PORCIONATO, M.A.F. et al. Efeito da fervura, resfriamento ou congelamento na qualidade do leite cru. Revista Acadêmica Ciências Agrárias e Ambientais, v.6, p.511-517, 2008.

RAMOS, M.; JUAREZ, M. Sheep milk. In: FUQUAY, J.W. et al. Encyclopedia of dairy sciences. 2.ed. United Kingdon: ELSEVIER, 2011. V.3, p.494-502.

SILVEIRA, P.R.; ABREU, L.R. Rendimento e composição físico-química do queijo prato elaborado com leite pasteurizado pelo sistema HTST e injeção direta de vapor. Ciência e Agrotecnologia, v.27,p.1340-1347,2003. Disponível em: $<$ http:// dx.doi.org/10.1590/S1413-70542003000600019>. Acesso em: 08 jan. 2013. doi: 10.1590/S1413-70542003000600019.

VIEIRA, C. et al. A produção vitivinícola da Serra Gaúcha Brasil e de Mendonza - Argentina: uma análise comparativa a luz das teorias de desenvolvimento regional. Revista Estudos do CEPE, v.26, p.30-56, 2007. Disponível em: <http://online. unisc.br/seer/index.php/cepe/article/view/405/236>. Acesso em: 08 jan. 2013.

WALSTRA, P. et al. Dairy science and technology. 2.ed. United States of America: TAYLOR \& FRANCIS, 2006. 763p.

ZHANG, R.H. et al. Effects of freezing on composition and fatty acid profiles of sheep milk and cheese. Small Ruminant Research, v.64, p.203-210, 2006. Disponível em: $<\mathrm{http}$ ://dx.doi.org.ez45.periodicos.capes.gov.br/10.1016/j. smallrumres.2005.04.025>. Acesso em: 16 mar. 2012. doi: 10.1016/j.smallrumres.2005.04.025. 